\title{
Formation of copper porous structures under near-equilibrium chemical vapor deposition
}

\author{
A.S. Kornyushchenko*, V.V. Natalich, V.I. Perekrestov \\ Sumy State University, Laboratory of Vacuum Nanotechnologies, 2, Rimsky-Korsakov Str., 40007 Sumy, Ukraine
}

\section{A R T I C L E I N F O}

\section{Article history:}

Received 31 August 2015

Received in revised form

25 February 2016

Accepted 26 February 2016

Communicated by: D.W. Shaw

Available online 4 March 2016

Keywords:

A1. Crystal morphology

A1. Nanostructures

A1. Nucleation

A3. Chemical vapor deposition processes

B1. Metals

B1. Nanomaterials

\begin{abstract}
A B S T R A C T
The mechanism of copper structure formation under near-equilibrium conditions in a chemically-active medium-condensate system has been investigated. The desired conditions have been implemented using CVD system. Copper chloride $\mathrm{CuCl}_{2}$ was used as a source material, and mixture of hydrogen with nitrogen served as a working gas. The influence of the evaporation temperature, condensation temperature and state of the growth surface on the porous structures formation has been investigated. It has been established, that the structure formation mechanism is determined by layer-by-layer or normal crystal growth, nucleation and growth of whiskers, and also by partial intergrowth of structural elements.
\end{abstract}

(c) 2016 Elsevier B.V. All rights reserved.

\section{Introduction}

Depending on structural and morphological characteristics porous structures and nanoparticles systems have great potential for application as catalysts [1-5], sensors [6-10], electrodes in electrochemical batteries [11-14], solar cells [15], fuel cells [16] and other active elements. Different methods are used with the purpose to form nanoparticles systems with uniform size and shape. Among them homogeneous nucleation from supersaturated vapors [17-22] and colloidal self-assembly [23,24]. Alumina membranes are widely used in order to form ordered nanoparticles arrays on the substrate [25-28].

One of the most widespread method of nanoporous metal structures formation is dealloying process, which consist in selective chemical or electrochemical etching of a desired component from its alloy [29-34]. Also porous metal nanosystems can be synthesized using a template with desired pore structure. After the cavities in the template are filled with a metal, the template material is removed $[35,36]$.

Thus, the conventional methods of metal nanosystems synthesis in the form of ordered nanoparticles or nanoporous structures utilize rather complicated multistage processes. Our previous works [37-40] have shown that, self-assembling of ordered nanoparticles system or

\footnotetext{
* Corresponding author.

E-mail address: ganna.korniushchenko@fulbrightmail.org (A.S. Kornyushchenko).
}

nanoporous structures can be implemented under near-equilibrium condensation conditions in the plasma-condensate system. It has been established, that the nucleation occurs only on the limited amount of active centers. In this case, the free energy minimization together with Ostwald ripening determine the monodispersity of the nanoparticles shape and size on the nucleation stage [41-44]. The more prolonged condensation results in the porous structures growth which is caused by an incomplete intergrowth of newly formed nanoparticles [45,37-39].

The analysis of literature reviews has shown that the technology of nanoporous metal structures self-assembling is at the early stage of its development. It is not mentioned even in quite recent reviews [46]. In order to provide an effective performance of the technologies operating near-equilibrium, tree problems have to be solved implementation of technological conditions for metals condensation at critically small supersaturations; realization of steady-state condensation conditions which allow to obtain reproducible structural and morphological characteristics; investigation of the interdependence between the process technological parameters and the structure formation mechanisms.

It is known that metals are bad-volatile substances having a low value of the equilibrium pressure. This fact complicates nearequilibrium conditions implementation. Obviously, under such conditions, the depositing vapor pressure should be of the same order as the equilibrium pressure. For the majority of metals (except $\mathrm{Mg}, \mathrm{Zn}, \mathrm{Cd}$ and $\mathrm{Hg}$ [47]) the equilibrium pressure does not exceed $10^{-5} \mathrm{~Pa}$ even for high temperatures. The realization of near-equilibrium conditions also requires deposition of extremely 
weak vapor fluxes. All these together, make it hard to control such weak fluxes and obtain reproducible results.

The equilibrium pressure can be increased conventionally by an increase in re-evaporation processes intensity. One solution is to influence by plasma onto the growth surface. In this case, the reevaporation is enhanced not only by plasma heating, but also by direct energy transfer from the plasma particles to adatoms. In our previous works $[38,45]$, this energy exchange was taken into account by the decrease in the desorption energy up to the effective value. It is known that, the equilibrium pressure value $P_{0}$ is determined by equation [48]:

$P_{0}=A\left(T_{c}\right) \exp \left(-\frac{E_{d}}{k_{B} T_{c}}\right)$,

here $A\left(T_{c}\right)$ is a coefficient which depends on the condensation temperature $T_{c}, k_{B}$ is Boltzmann constant, $E_{d}$ is the desorption energy.

Eq. (1) follows that a decrease in the desorption energy to the effective value leads to an increase in the equilibrium pressure value. Therefore, the plasma action onto the growth surface allows to deposit more intense and as a result more controlled vapor fluxes even at low supersaturations. In such a way the stationarity of the deposition process can be increased. In this context, a question arises how the chemically active medium influences the system approaching to the equilibrium. In [38] we have suggested that weakly bounded to the growth surface adatoms may react with other chemical elements and return to vapor state. For example, the adsorbed carbon atoms can be bonded with hydrogen and transform into more volatile hydrocarbon fraction which vaporizes at relatively low temperatures.

In order to provide near-equilibrium conditions for low-volatile substances, chemically active media such as chemically active gases should be used. In this case, re-evaporation of adatoms weakly bound to the growth surface is stimulated by reverse chemical reactions. In other words, the usage of the chemically active medium provides removal of adatoms which are weakly bound with the growth surface, providing selective condensation exclusively on active centers. The influence of the active medium leads to a decrease of the desorption energy up to effective value, and stimulates the approach of the system active mediumcondensate to thermodynamic equilibrium conditions.

Quite often similar structures are obtained using absolutely different technologies utilizing plasma-condensate or chemically active medium-condensate systems. An example would be formation of different carbon molecular forms using CVD or PVD technology under near-equilibrium conditions [49-51]. Such similarities in the structures can be explained by identical thermodynamic conditions, which in turn are determined by the same values of the chemical potential difference of atoms in vapor and condensed state.

That is why, the aim of the work, firstly, is to study on the example of copper the nucleation and the growth mechanisms of condensates formation under near-equilibrium conditions implemented in the chemically active medium-condensate system. Secondly, to compare peculiarities of condensate formation mechanisms with our previous results obtained for the plasmacondensate system. We believe that this study will broaden significantly understanding of underlying physical process at condensation under near-equilibrium conditions.

In our previous works $[44,52,53]$ the nucleation and growth of copper layers under near-equilibrium conditions in plasmacondensate system have already been studied. That is why copper has been selected in this work as a material for comparative analysis. It is necessary to point out, that the choice of copper is also caused by wide application of copper-based porous nanosystems as catalytic materials and biosensors [54-59]. Besides that, during the condensation process copper reacts badly with chemically active residual gases as compared to other metals. This fact is very important at low deposition rates. Thus, according to $[60,61]$ pure copper layers can be formed under near-equilibrium conditions even in oxygen medium. The negligible influence of chemically active gases will allow to understand better peculiarities of the condensation process.

\section{Methodology of the experiment}

\subsection{Materials}

It is known, that decomposition of metallo-organic compounds is often used in order to synthesize metals by CVD. But these metallo-organic precursors are often unstable and can contaminate the resulting layer with carbon and oxygen. One of widespread methods of metals formation by CVD is usage of hydrogen reduction reaction from chlorides. In the case of copper, deposition can be carried out by the hydrogen reduction of the copper I chloride or copper II chloride. In [62] the following reduction reaction is given for CVD copper formation:

$$
\mathrm{CuCl}_{2} \text { (powder) }+\mathrm{H}_{2} \text { (gas) } \rightarrow \mathrm{Cu} \text { (solid) }+2 \mathrm{HCl} \text { (gas) }
$$

In the article [63] the table of known copper precursors for CVD is presented. Among the all precursors both copper I chloride and copper II chloride are listed. Thus, $\mathrm{CuCl}_{2}$ and $\mathrm{CuCl}$ both are wellknown precursors.

But $\mathrm{CuCl}_{2}$ is one of the most widespread copper compounds after copper sulfite. It's melting temperature is $498^{\circ} \mathrm{C}$ and boiling temperature $993{ }^{\circ} \mathrm{C}$. At $1000{ }^{\circ} \mathrm{C}$ copper II chloride decomposes into $\mathrm{CuCl}$ and $\mathrm{Cl}_{2}$. For comparison, $\mathrm{CuCl}$ has melting point at $426{ }^{\circ} \mathrm{C}$ and boiling point at $1490{ }^{\circ} \mathrm{C}$. In our work, the problem was to create extremely week copper deposition rates. That is why, we have chosen $\mathrm{CuCl}_{2}$ as a precursor, because this compound has less amount of copper. As a source $\mathrm{CuCl}_{2}$ 99.999\% purity from the company ABCR was used, the mass of the substance used for one experiment was 8-10 g.

Silicon wafers with $\langle 111\rangle$ orientation were used as substrates. It is known, that under near-equilibrium conditions the structure formation mechanism depends greatly on the growth surface state. That is why silicon substrates with both polished and rough (treated with a sandpaper) surface were used.

\subsection{Methods}

A self-made chemical vapor deposition (CVD) system was used in order to form copper layers. The schematic representation of the system is given in Fig. 1. The main element of the setup is a quartz tube which can sustain high heating temperatures. A tube of smaller diameter having narrowed end was installed inside in order to form a directed flow of a carrier gas. A mixture of hydrogen and nitrogen in ratio 1:3 was used as the carrier gas. The speed of the gas inlet was controlled by means of the flowmeter. The pressure control was installed with purpose to maintain a desired pressure. The vacuum conditions have been created by means of a turbo pump. The CVD setup has three temperature zones identified in Fig. 1 as zone 1, zone 2, zone 3. Different

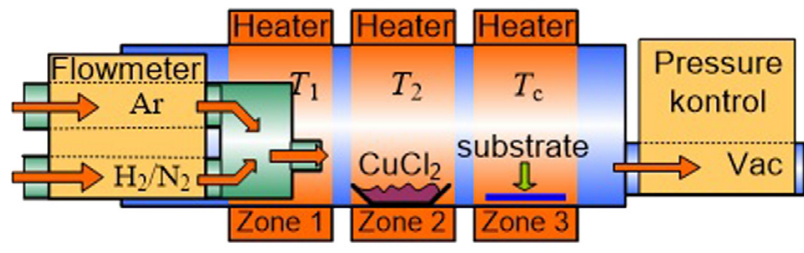

Fig. 1. Schematic representation of CVD device. 
temperature values $T_{1}, T_{2}$ and $T_{c}$ can be maintained in each zone separately with help of the heaters.

The reduction reaction (2) was used in the technological process of copper layers formation. A cuvette with an open top was filled with the copper II chloride powder and placed in the temperature zone 2 (evaporation zone). Evaporated copper II chloride reacted with hydrogen. Copper has been deposited on the substrate positioned in the temperature zone 3 (condensation zone). Hydrogen chloride was removed from the condensation area with the carrier gas flow.

On the first stage of the technological process, the quartz tube was pumped off to the pressure level $10^{-5} \mathrm{~Pa}$. Then high purity argon was introduced into the working area up to the pressure $7500 \mathrm{~Pa}$ and heating was started. The argon was used with purpose of the reduction reaction suppressing until the system reaches required temperature regime. When the temperatures in all three zones have reached desired values, argon was pumped out and the working gas mixture $\mathrm{H}_{2}+\mathrm{N}_{2}$ was introduced into the tube. This moment is considered as the beginning of the deposition process. During the deposition process the $\mathrm{H}_{2}+\mathrm{N}_{2}$ mixture continuously passed through the reactor and simultaneous pumping maintained pressure at the level 10,000 $\mathrm{Pa}$.

Relatively low evaporation temperatures of $\mathrm{CuCl}_{2}\left(T_{2}=200\right.$, $350^{\circ} \mathrm{C}$ ) were used in order to implement near-equilibrium condensation conditions. Also extremely small copper deposition rates were achieved by adjusting such technological parameters as the condensation temperature $T_{c}$, the carrier gas flow rate $W$ and the working gas pressure $P$. After the deposition process completed, the heating was turned off. Then the working area was pumped off and argon was introduced up to pressure 50, $000 \mathrm{~Pa}$. The system was kept in this state until complete cooling down.

\subsection{Instrumentals}

The structure and composition of copper condensates were investigated by scanning electron microscopy equipped with energy dispersive X-ray elemental analysis (SEM, FEI NanoSEM 230) and Xray diffraction (XRD, SIEMENS (2x) D5000 X-ray diffractometer).

The layers porosity was determined using following methodology; first, the substrate mass $\left(m_{1}\right)$ was measured before the deposition. Then, after condensation completed, the mass of the layer and substrate together $\left(m_{2}\right)$ was measured. The layers thickness $d_{s}$ was determined from SEM cross-section images. The volume of deposited copper layer $V_{C u}$ can be calculated using following relation:

$V_{C u}=\frac{m_{2}-m_{1}}{\rho_{C u}}$

here $\rho_{C u}$ is density of copper. The porous layer volume can be determined as:

$V_{c}=S d_{s}$

where $S$ is the porous layer area. Then, the layer porosity can be found as follows:

$\beta_{b}=100 \% \frac{V_{c}-V_{C u}}{V_{c}}=100 \%\left(1-\frac{m_{2}-m_{1}}{\rho_{C u} S d}\right)$

\section{Results and discussion}

Four sets of experiments have been performed in order to investigate copper structure formation mechanisms under nearequilibrium conditions in a chemically-active medium-condensate system. The deposition parameters of all experiments are presented in the Table 1. It is necessary to point out, that copper was deposited simultaneously on both mirror polished and rough silicon substrates under the similar technological conditions. The experiments have shown, that the state of the growth surface influences significantly the mechanisms of copper nucleation and growth. At this point, depending on the condensation temperature, a layer-by-layer or a normal crystal growth mechanism can be realized. These two mechanisms correspond to adatoms embedding into an atomically flat or an atomically rough growth surface, respectively. The crystal faceting formation is a characteristic of the layer-by-layer growth mechanism. From the other hand, the normal growth is characterized by round shaped crystals formation [64].

Fig. 2 gives structure of copper layers obtained under technological condition of the first experimental series performed on

Table 1

Technological parameters of copper deposition.

\begin{tabular}{lllllll}
\hline $\begin{array}{l}\text { Number of experi- } \\
\text { mental set }\end{array}$ & $\begin{array}{l}W, \mathrm{~cm}^{3} / \\
\min \end{array}$ & $P$, Pa & $\begin{array}{l}\text { Deposition } \\
\text { time, h }\end{array}$ & $\begin{array}{l}T_{1}, \\
{ }^{\circ} \mathrm{C}\end{array}$ & $\begin{array}{l}T_{2}, \\
{ }^{\circ} \mathrm{C}\end{array}$ & $\begin{array}{l}T_{c}, \\
{ }^{\circ} \mathrm{C}\end{array}$ \\
\hline 1 & 15.35 & 10,000 & 2 & 350 & 350 & 350 \\
2 & 15.35 & 10,000 & 2 & 350 & 350 & 500 \\
3 & 15.35 & 10,000 & 2 & 200 & 200 & 200 \\
4 & 15.35 & 10,000 & 2 & 200 & 200 & 300 \\
\hline
\end{tabular}
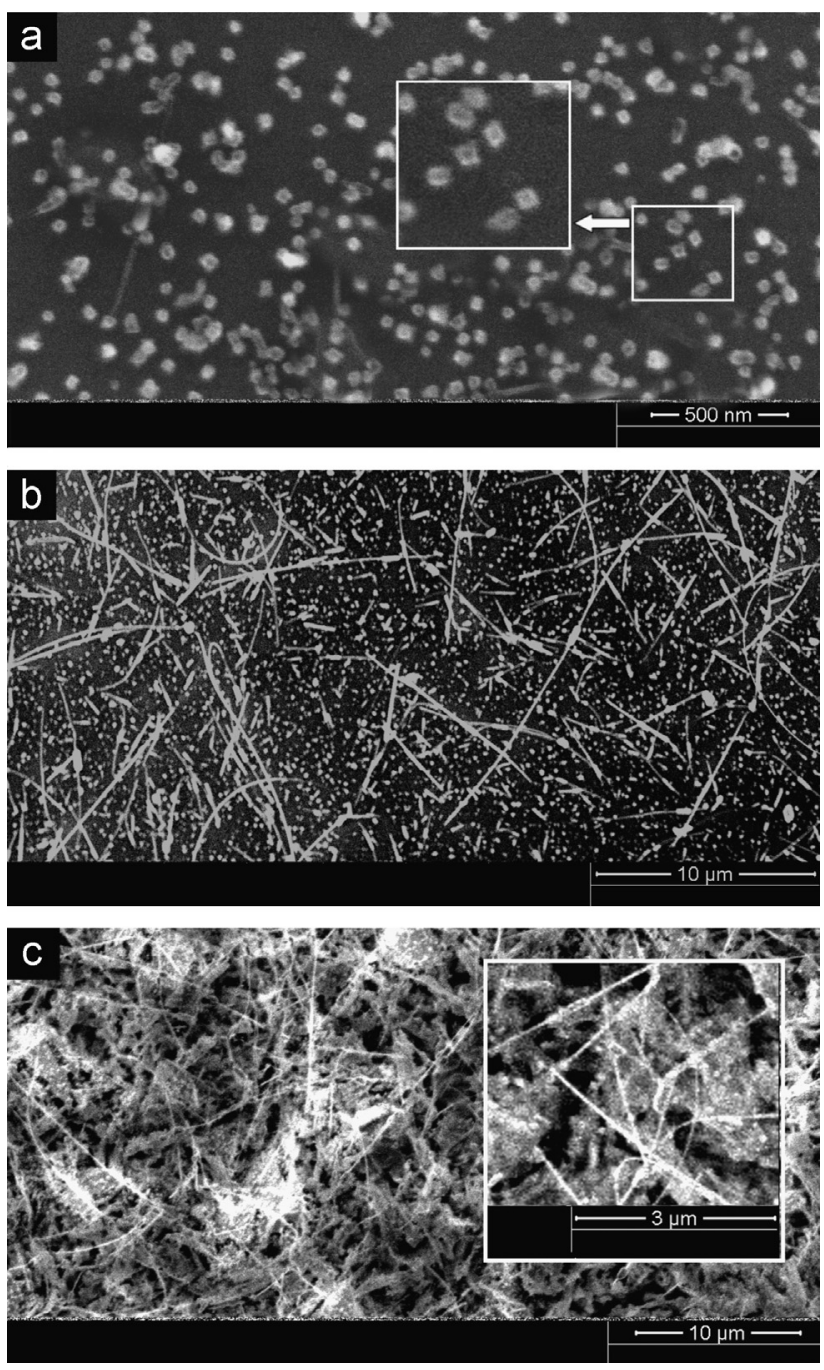

Fig. 2. The layer-by-layer growth of copper implemented at the temperatures $T_{1}=T_{2}=T_{c}=350{ }^{\circ} \mathrm{C}$ on the polished ( $\mathrm{a}, \mathrm{b}$ the same structure and different magnifications) and rough (c) silicon surface. 
both polished and rough silicon substrates. From SEM images made at different magnifications (Fig. 2a and b) a conclusion can be made about extremely low rates of copper condensation on the polished silicon surface. This fact confirms nearness to thermodynamic equilibrium condensation conditions. The crystal faceting and whiskers for.

The transition from the polished to the rough growth surface under the same technological conditions leads to a significant increase in the copper layer thickness. At the same time, the condensate structure is the same (Fig. 2c). In this case, a highly porous layer is formed consisting of interconnected whiskers and nanoparticles. It is obvious, that more intense crystal growth on the rough growth surface is determined be higher active centers concentration. This fact is a prerequisite for the additional active centers formation and leads to more effective homonucleation of new nanoparticles and whiskers.

Under technological parameters of the second experimental set and use of polished silicon substrates, relatively large clusters form only on selected areas of the substrate (Fig. 3a). Such selective growth is explained by the preferable nucleation only on limited amount of active centers under Volmer-Weber conditions. The clusters have polycrystalline structure, as a result of rather effective secondary nucleation on already formed copper crystals.

At the identical technological conditions, the rough growth surface usage provides a significant increase in the amount of condensed matter. In this case, the porous structure formed consists of weakly connected with each-other faceted crystals (Fig. 3b). It is necessary to point out, that the increase in the condensation temperature from $350{ }^{\circ} \mathrm{C}$ leads to a gradual decrease in the whiskers amount. At the temperatures $500{ }^{\circ} \mathrm{C}$ and higher, their formation is not observed at all.

The experimental sets three and four by which the predominant normal crystal growth is implemented deserve a particular attention. At this point atoms embed into atomically rough growth surface. The transition to the normal structure formation mechanism is observed at a decrease in the temperatures in all three zones up to $200{ }^{\circ} \mathrm{C}$. The characteristic feature of this
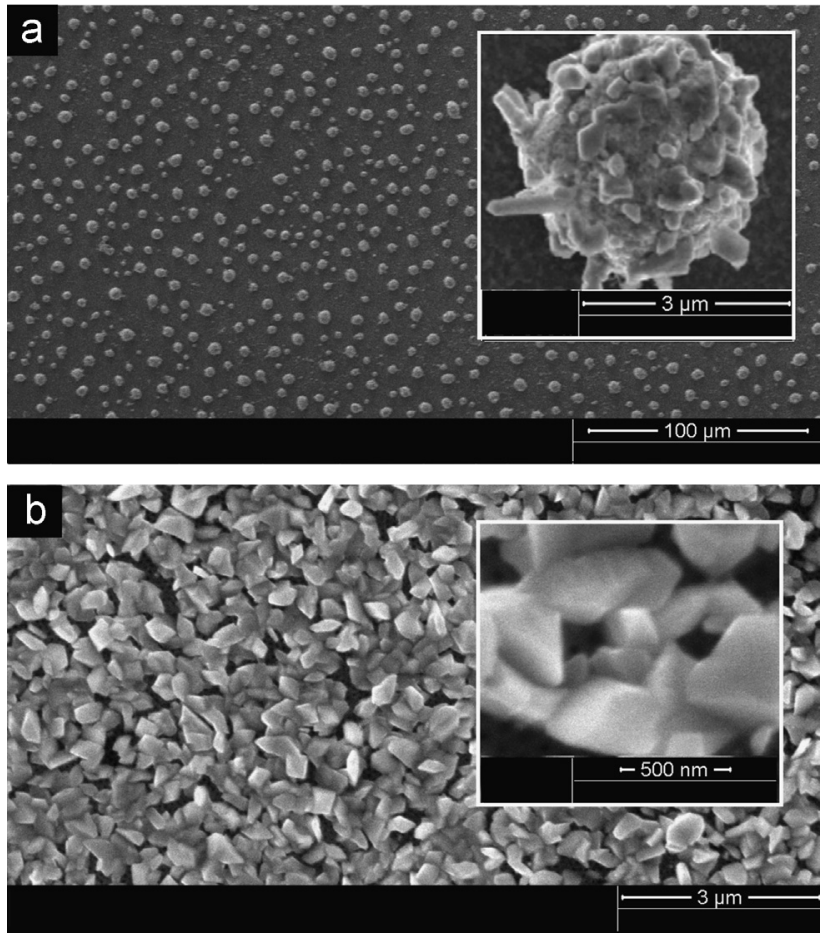

Fig. 3. The morphology of copper faceted condensates on the polished (a) and rough (b) silicon surface at $T_{1}=T_{2}=350{ }^{\circ} \mathrm{C}$ and $T_{c}=500{ }^{\circ} \mathrm{C}$. mechanism is round crystals shape. Fig. 4 gives examples of such structures obtained on the polished ( $\mathrm{a}$ and $\mathrm{b}$ ) and rough (c) silicon surface. The same as in previous cases, the crystals growth rate is significantly higher for the rough surface. At the same time, the decrease in the condensation temperature from 300 to $200{ }^{\circ} \mathrm{C}$ leads to complete disappearance of the crystal faceting and averaging of the structural elements size (Fig. 4a and b).

The same as for the plasma-condensate system mentioned before, the self-organization of crystals identical size is due to Ostwald ripening process, which intensifies at a decrease in the depositing vapors supersaturation. From the other hand, according to Eq. (1), the equilibrium pressure should decrease at an increase in the condensation temperature $T_{c}$ and at all the other conditions being the same it is a prerequisite for the supersaturation increase. This contradiction can be explained by the decrease in the adatoms desorption energy $E_{d}$ up to the effective value. The mechanism of this decrease is determined by approaching of the copper reduction reaction (2) to equilibrium conditions. In this case, due to chlorine content excess, the reverse to reduction chemical process can take place which consist in formation of more volatile copper compounds such as $\mathrm{CuCl}$ and $\mathrm{CuCl}_{2}$ and their following evaporation.
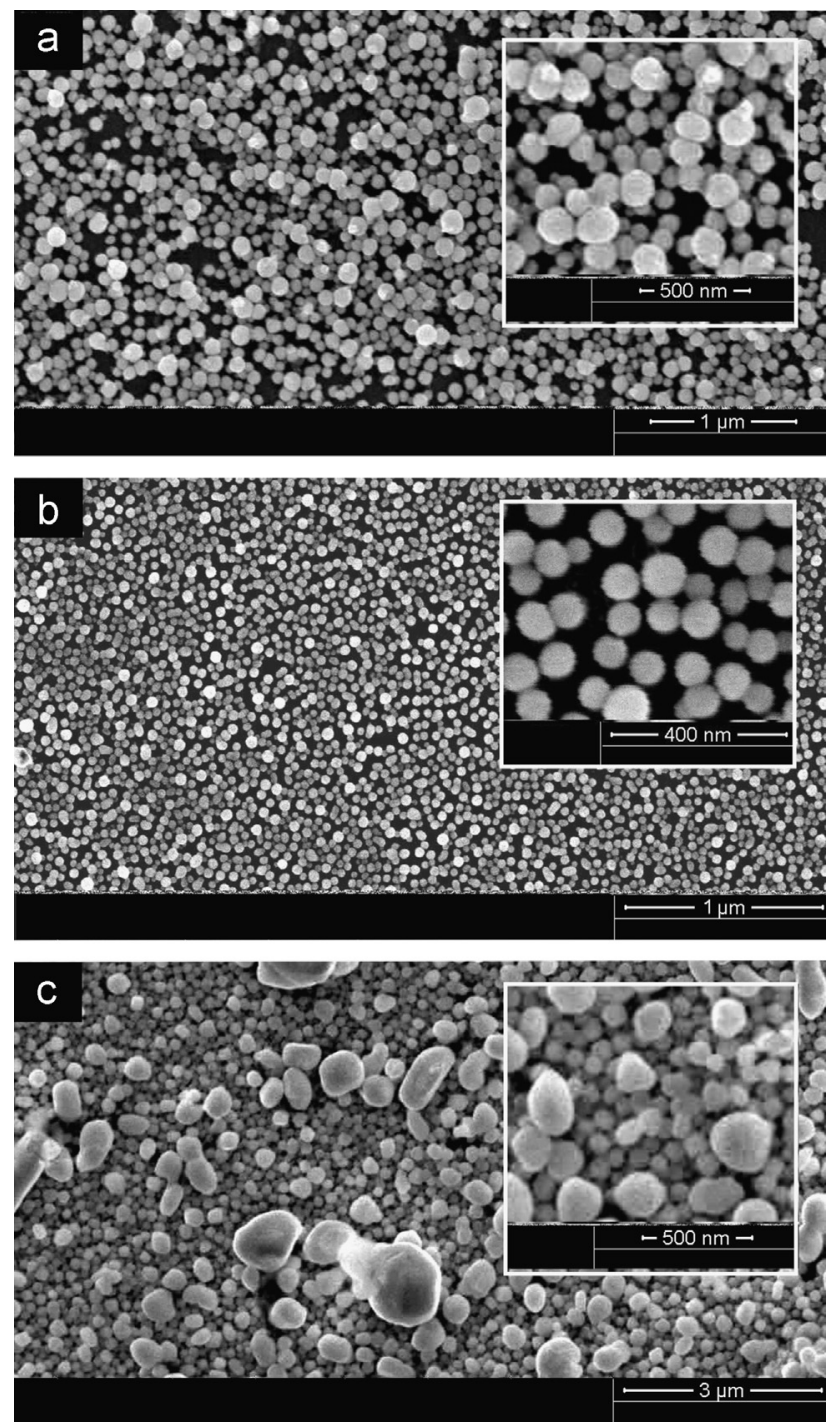

Fig. 4. The morphology of copper round shaped condensates formed at $T_{1}=T_{2}=200{ }^{\circ} \mathrm{C}$ on the polished $\left(\mathrm{a}-T_{c}=300{ }^{\circ} \mathrm{C}, \mathrm{b}-T_{c}=200{ }^{\circ} \mathrm{C}\right)$ and rough $(\mathrm{c}-$ $T_{c}=200{ }^{\circ} \mathrm{C}$ ) silicon surface. 
A more rigorous explanation of the change of the structure formation mechanism can be given in terms of the variation in a monatomic step free energy $\alpha_{i}$. In the simplest case, it is determined by equation [64]:

$\alpha_{i}=n\left(E-k_{B} T_{c} \ln (1+2 \chi)\right)$

here $\chi=\exp \left(-E / k_{B} T_{c}\right), n$ is the density of atoms on the end face of the monatomic step, $E$ is bonding energies of copper atoms on the monatomic step. At transition from the atomically flat (Fig. $3 \mathrm{~b}$ ) to the atomically rough growth surface (Fig. $4 \mathrm{a}$ and b), the monatomic step free energy $\alpha_{i}$ becomes zero. Analysis of Eq. (3) shows, that the decrease of $\alpha_{i}$ up to zero is observed at an increase in $T_{c}$ and decrease in $E_{1}$. The experimental results have shown, that in our case the transition to the atomically rough growth surface occurs at the decrease in the condensation temperature $T_{c}$. It is explained by significant decrease in $E$ up to effective value due to the reverse chemical processes intensification.

The energy dispersive X-ray elemental analysis shows presence of oxygen in the copper condensates with concentration from 1.4 to 3.5 at\%. In the majority of the condensates formed on the polished and rough surface oxygen concentration is the same, and is approximately of about 2 at\% (Fig. 5). But with an increase in the porosity and thickness, oxygen concentration rises up to 3.4 at\%. In other words, the concentration of oxygen at the top layer is bigger than near the substrate surface. That is why it can be concluded, that presence of oxygen in the condensate is mainly determined by water adsorption and by uppermost layer oxidation after the technological setup opening. The example of such highly porous structure is represented in Fig. 2c.
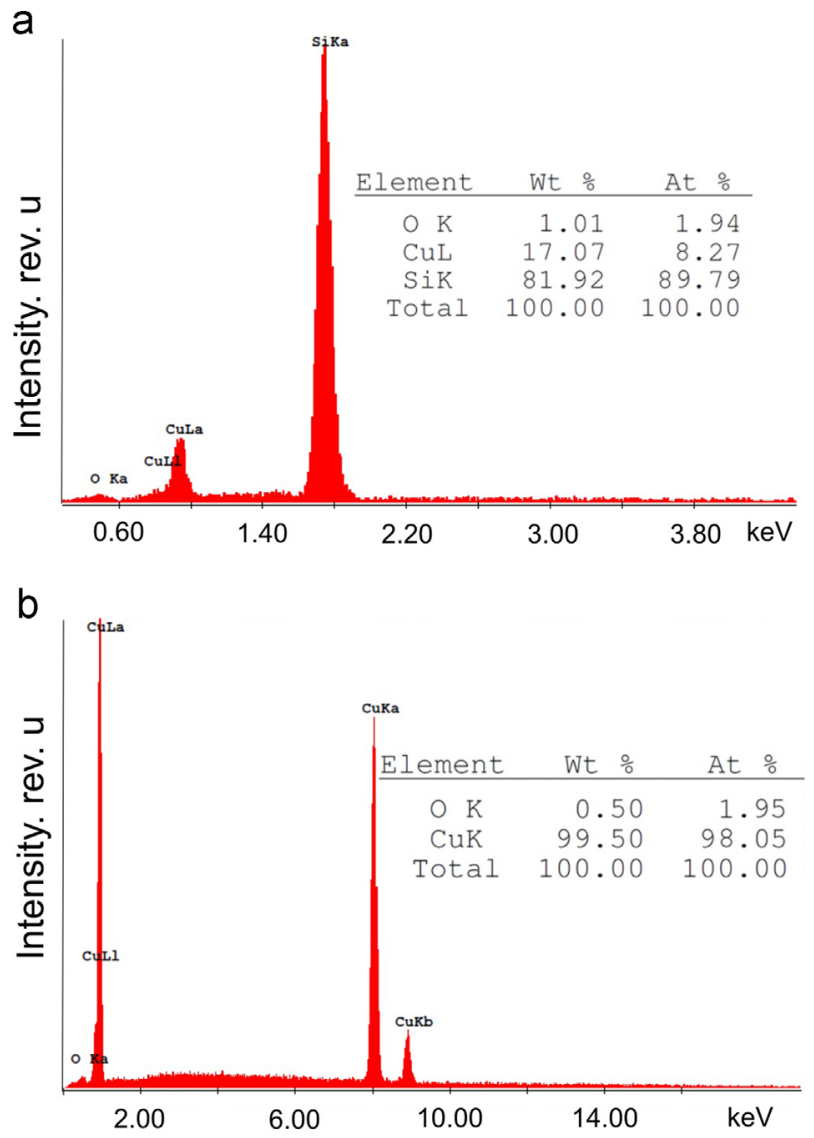

Fig. 5. The elemental analysis results of copper formed on the polished ( $\mathrm{a}-$ the structure is given in Fig. 3a) and rough (b - the structure is given in Fig. 3b) silicon surface.

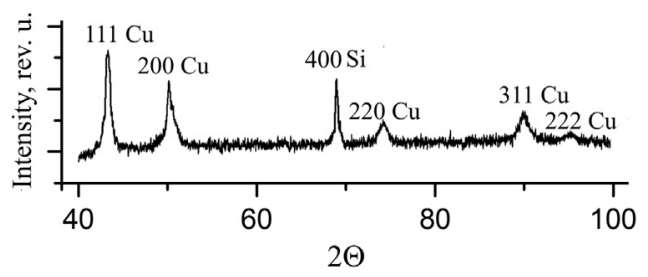

Fig. 6. The XRD pattern of copper layer formed on the rough silicon surfaces (the structure is given in Fig. $3 \mathrm{~b}$ ).

From the other hand oxygen presence in the condensate can be caused by its capture from residual gases during copper condensation. The elemental analysis of the substrate has shown that for the rough growth surface oxygen level can reach from 1.3 to 1.6 at\%.

Finally, the condensates phase composition has been investigated. In this case, the analysis of X-ray diffraction patterns has shown that all diffraction maxima correspond to copper. Silicon pick can also be seen. Fig. 6 gives a typical diffraction pattern. The period of crystalline fcc lattice calculated based on the diffraction patterns is $0.3615 \mathrm{~nm}$. This value is in good agreement with the copper lattice parameters.

The described above structure formation mechanisms under near-equilibrium conditions in the chemically active mediumcondensate system to the great extent correspond to the structure formation mechanisms in the plasma-condensate system $[44,52,53]$. For both technological solutions, near-equilibrium condensation allows to implement the transition from layer-bylayer to the normal growth of round shaped crystals together with self-organization of their identical size due to Ostwald ripening. Besides that, the porous structures consisting of weakly bound faceted crystals [53] and round shaped crystals [44,52] can be grown realizing both of these two methods. At the same time, whiskers grow more effectively using CVD technology, but in the plasma-condensate system more morphologically diverse structures with less oxygen content can be formed [53].

Using relation (5) the layers porosity has been calculated. For condensates with structures given in Figs. $2 \mathrm{c}$ and $3 \mathrm{~b}$ the porosity values found to be $65 \%$ and $48 \%$, correspondingly for layer's thicknesses from 3.5 to $3.7 \mu \mathrm{m}$.

\section{Conclusions}

1. The possibility of different porous structures formation has been established using near-equilibrium conditions in the chemically active medium-condensate system on the example of copper. The structure formation mechanism is based on such processes as the layer-by-layer or the normal crystal growth, the nucleation and growth of whiskers, and the incomplete intergrowth of the structural elements. In this case, the transition from the layer-by-layer to the normal crystals growth is mainly caused by the decrease in copper adatoms binding energy up to the effective value due to the reverse chemical reactions.

2. The low deposition rates observed for the polished silicon substrates evidence about extremely weak supersaturations of the depositing vapors. The transition to higher deposition rates in case of the rough silicon surface indicates the presence of large amount of active centers for the condensate nucleation. As a result, more effective secondary homonucleation on the already formed copper crystals occurs in this case.

3. From our point of view, the reduction reaction and subsequent homonucleation of copper clusters preferably occurs on the substrate surface. It is known, that thermally isotropic surface 
serves as catalyst for chemical reaction initiation. It can be explained by the fact, that probability of adsorbed atoms interaction is higher in the case of their diffusive movement on the substrate surface than in the volume at their motion with directed carrier gas flow. The different deposition rates on the polished and rough growth surface confirm that the reduction reaction do takes place on the substrate. The change of condensate morphology during the deposition process also evidences that the reaction occurs on the surface. For example, in the first set of experiments the layers nucleate in the form of faceted crystals. Then, during more prolonged condensation, whiskers start to grow (Fig. 3). In the case of homonucleation in volume, the condensates must have structure homogeneous with respect to thickness.

4. The comparative analysis of the structural and morphological characteristics of copper condensates obtained with help of the chemically active medium-condensate system and the plasmacondensate system has shown the similar growth mechanism. The both technological solutions allow to form round shaped crystals of approximately identical size, which is result of the normal growth and Ostwald ripening. Besides that, the porous structures formation in both cases is a result of the incomplete crystals intergrowth which were selectively nucleated only on active centers. Consequently, the main prerequisite for porous structures formation or system of round shaped crystals formation is realization of near-equilibrium conditions. It is necessary to point out, that the method of implementation of these conditions does not matter. At the same time, the mechanism of approaching to thermodynamic equilibrium for both methods is explained by the decrease in the desorption energy up to the effective value.

\section{Acknowledgments}

This work was supported by Ministry of Science and Education of Ukraine within the project "Formation of metals porous microand nanosystems on the basis of interrelated dissipative and conservative self-organization processes".

\section{References}

[1] T.-J. Kuo, C.-N. Lin, C.-L. Kuo, M.H. Huang, Growth of ultralong ZnO nanowires on silicon substrates by vapor transport and their use as recyclable photocatalyst, Chem. Mater. 19 (21) (2007) 5143-5147.

[2] K. Wegner, H.C. Ly, R.J. Weiss, S.E. Pratsinis, A. Steinfeld, In situ formation and hydrolysis of $\mathrm{Zn}$ nanoparticles for $\mathrm{H}_{2}$ production by the 2-step $\mathrm{ZnO} / \mathrm{Zn}$ watersplitting thermochemical cycle, Int. J. Hydrog. Energy 31 (1) (2006) 55-61.

[3] V. Bansal, H. Jani, J. Du Plessis, P.J. Coloe, S.K. Bhargava, Galvanic replacemen reaction on metal films: a one-step approach to create nanoporous surfaces for catalysis, Adv. Mater. 20 (4) (2008) 717-723.

[4] Zh.-T. Liu, X. Li, Zh.-W. Liu, J. Lu, Synthesis and catalytic behaviors of cobalt nanocrystals with special morphologies, Powder Technol. 189 (3) (2009) 514-519.

[5] Y. Liang, P. Wang, H.-B. Dai, Hydrogen bubbles dynamic template preparation of a porous $\mathrm{Fe}-\mathrm{Co}-\mathrm{B} / \mathrm{Ni}$ foam catalyst for hydrogen generation from hydrolysis of alkaline sodium borohydride solution, J. Alloy. Compd. 491 (1-2) (2010) 359-365.

[6] A. Wei, L. Pan, W. Huang, Recent progress in the $\mathrm{ZnO}$ nanostructure-based sensors, Mater. Sci. Eng. B 176 (18) (2011) 1409-1421.

[7] A.K.M. Kafi, A. Ahmadalinezhad, J. Wang, D.F. Thomas, A. Chen, Direct growth of nanoporous Au and its application in electrochemical biosensing, Biosens. Bioelectron. 25 (11) (2010) 2458-2463.

[8] N. Wang, K. Burugapalli, W. Song, J. Halls, F. Moussy, A. Ray, Y. Zheng, Electrospun fibro-porous polyurethane coatings for implantable glucose biosensors, Biomaterials 34 (4) (2013) 888-901.

[9] A. Abburi, N. Abrams, W.J. Yeh, Synthesis of nanoporous platinum thin films and application as hydrogen sensor, J. Porous Mater. 19 (5) (2012) 543-549.

[10] S.A.G. Evans, J.M. Elliott, L.M. Andrews, P.N. Bartlett, P.J. Doyle, G. Denuault, Detection of hydrogen peroxide at mesoporous platinum microelectrodes, Anal. Chem. 74 (6) (2002) 1322-1326.
[11] X.H. Huang, X.H. Xia, Y.F. Yuan, F. Zhou, Porous ZnO nanosheets grown on copper substrates as anodes for lithium ion batteries, Electrochim. Acta 56 (14) (2011) 4960-4965.

[12] X.G. Zhang, Fibrous zinc anodes for high power batteries, J. Power Sour. 163 (1) (2006) 591-597.

[13] X. Tao, F. Chen, Y. Xia, H. Huang, Y. Gan, X. Chen, W. Zhang, Decoration of sulfur with porous metal nanostructures: an alternative strategy for improving the cyclability of sulfur cathode materials for advanced lithium-sulfur batteries, Chem. Commun. 49 (2013) 4513-4515.

[14] C. Yang, D. Zhang, Y. Zhao, Y. Lu, L. Wang, J.B. Goodenough, Nickel foam supported Sn-Co alloy film as anode for lithium ion batteries, J. Power Sour. 196 (24) (2011) 10673-10678.

[15] A. Hagfeldt, G. Boschloo, L. Sun, L. Kloo, H. Pettersson, Dye-sensitized solar cells, Chem. Rev. 110 (11) (2010) 6595-6663.

[16] W. Yuan, Y. Tang, X. Yang, Zh Wan, Porous metal materials for polymer electrolyte membrane fuel cells, Appl. Energy 94 (2012) 309-329.

[17] X.C. Jiang, Q.H. Zeng, C.Y. Chen, A.B. Yu, Self-assembly of particles: some thoughts and comments, J. Mater. Chem. 21 (2011) 16797-16805.

[18] R.B. Wehrspohn, Ordered porous nanostructures and applications, in: David J. Lockwood (Ed.) Nanostructure Science and Technology, Springer, US, 2005.

[19] B. Vigolo, R. Mafouana, C. Goyhenex, J. Faerber, J. Arabski, C. Hirlimann, J.L. Rehspringer, Self-controlled growth and two-dimensional ordering of metallic nanoparticles, Appl. Phys. Lett. 88 (2006) 153122-153124.

[20] K. Siskova, K. Safarova, J.H. Seo, R. Zboril, M. Mashlan, Non-chemical approach toward 2D self-assemblies of Ag nanoparticles via cold plasma treatment of substrates, Nanotechnology 22 (27) (2011) 275601-275608.

[21] L. Qiang, D. Weimin, Large-scale synthesis and catalytic properties of nearly monodispersive nickel 3D nanostructures, Rare Met. Mater. Eng. 38 (12) (2009) 2080-2084.

[22] A. Ghadimi, L. Cademartiri, U. Kamp, G.A. Ozin, Plasma within templates: molding flexible nanocrystal solids into multifunctional architectures, Nano Lett. 7 (12) (2007) 3864-3868.

[23] J.H. Lee, Q. Wu, W. Park, Metal nanocluster metamaterial fabricated by the colloidal self-assembly, Opt. Lett. 34 (4) (2009) 443-445.

[24] L. Pena, M. Varon, Z. Konstantinovic, L. Balcellsrticles, Large 2D self-assembled domains of cobalt nanoparticles onto silicon wafers, J. Mater. Chem. 21 (2011) 16973-16977.

[25] Y. Lei, W. Cai, G. Wilde, Highly ordered nanostructures with tunable size, shape and properties: a new way to surface nano-patterning using ultra-thin alumina masks, Prog. Mater. Sci. 52 (4) (2007) 465-539.

[26] Y. Lei, S. Yang, M. Wu, G. Wilde, Surface patterning using templates: concept, properties and device applications, Chem. Soc. Rev. 40 (3) (2011) 1247-1258.

[27] S. Yang, F. Xu, S. Ostendorp, G. Wilde, Template-confined dewetting process to surface nanopatterns: fabrication, structural tunability, and structure-related properties, Adv. Funct. Mater. 21 (13) (2011) 2446-2455.

[28] V. Perekrestov, A. Kornyushchenko, V. Natalich, S. Ostendorp, G. Wilde, Formation of porous nickel nanosystems using alumina membranes as templates for deposition, Mater. Lett. 153 (2015) 171-174.

[29] M. Li, Y. Zhou, H. Geng, Fabrication of nanoporous copper ribbons by dealloying of Al-Cu alloys, J. Porous Mater. 19 (5) (2012) 791-796.

[30] H.B. Lu, Y. Li, F.H. Wang, Synthesis of porous copper from nanocrystalline twophase Cu-Zr film by dealloying, Scr. Mater. 56 (2) (2007) 165-168.

[31] V. Pugh, A. Dursun, S.G. Corcoran, Electrochemical and morphological characterization of Pt-Cu dealloying, J. Electrochem. Soc. 152 (11) (2005) B455-B459.

[32] Y. Ding, J. Erlebacher, Nanoporous metals with controlled multimodal pore size distribution, J. Am. Chem. Soc. 125 (26) (2003) 7772-7773.

[33] J.F. Huang, I.W. Sun, Fabrication and surface functionalization of nanoporous gold by electrochemical alloying/dealloying of $\mathrm{Au}-\mathrm{Zn}$ in an ionic liquid, and the selfassembly of l-cysteine monolayers, Adv. Funct. Mater. 15 (6) (2005) 989-994.

[34] A.J. Smith, D.L. Trimm, The preparation of skeletal catalysts, Annu. Rev. Mater. Res. 35 (2005) 127-142.

[35] K.M. Kulinowski, P. Jiang, H. Vaswani, V.L. Colvin, Porous metals from colloidal templates, Adv. Mater. 12 (11) (2000) 833-838.

[36] A. Walcarius, Mesoporous materials and electrochemistry, Chem. Soc. Rev. 42 (2013) 4098-4140.

[37] V.I. Perekrestov, Yu.O. Kosminska, A.A. Mokrenko, I.N. Kononenko, A. S. Kornyushchenko, Structure formation mechanisms of low-dimensional porous titanium systems condensed under quasi-equilibrium steady-state conditions, Vacuum 86 (1) (2011) 111-118.

[38] V.I. Perekrestov, A.I. Olemskoi, Yu.O. Kosminska, A.A. Mokrenko, Selforganization of quasi-equilibrium steady-state condensation in accumulative ion-plasma devices, Phys. Lett. A 373 (37) (2009) 3386-3391.

[39] V.I. Perekrestov, A.I. Olemskoi, A.S. Kornyushchenko, Yu.A. Kosminskaya, Selforganization of plasma-condensate quasi-equilibrium systems, Phys. Solid State 51 (5) (2009) 1060-1067.

[40] V.I. Perekrestov, A.A. Mokrenko, Yu. A. Kosminskaya, D.I. Rubets, Formation of nickel extended surface upon quasi-equilibrium steady-state condensation, J. Surf. Investig. 5 (4) (2011) 667-671.

[41] J. Tang, P. Photopoulos, A. Tserepi, D. Tsoukalas, Two-dimensional nanoparticle self-assembly using plasma-induced Ostwald ripening. Nanotechnology 22 (2011) 235306-235310.

[42] M. Zinke-Allmang, L.C. Feldman, M.H. Grabow, Clustering on surfaces, Surf. Sci. Rep. 16 (8) (1992) 377-463.

[43] P.L. Redmond, A.J. Hallock, L.E. Brus, Electrochemical Ostwald ripening of colloidal Ag particles on conductive substrates, Nano Lett. 5 (1) (2005) 131-135. 
[44] V.I. Perekrestov, Y.O. Kosminska, A.S. Kornyushchenko, V.M. Latyshev, Selforganization of copper nanosystems under Volmer-Weber conditions during quasi-equilibrium condensation, Physica B 411 (2013) 140-148.

[45] V.I. Perekrestov, A.S. Kornyushchenko, V.V. Natalich, Formation of chromium layers under Volmer-Weber conditions at critically small supersaturations, Solid State Sci. 33 (2014) 12-18.

[46] Y. Ding, Z. Zhang, in: R. Vajtai (Ed.), Springer Handbook of Nanomaterials, Springer-Verlag Berlin Heidelberg, 2013, pp. 779-818.

[47] R. Glang, in: L.I. Maissel, R. Glang (Eds.), Handbook of Thin Film Technology, 1, McGraw Hill Hook Company, New York, 1970, pp. 9-174.

[48] P. Zhang, X. Zheng, S. Wu, D. He, A computer simulation of nucleation and growth of thin films, Comput. Mater. Sci. 30 (3-4) (2004) 331-336.

[49] N. Zhao, Q. Cui, C. He, C. Shi, J. Li, H. Li, X. Du, Mater. Sci. Eng. A 460-461 (2007) $255-260$

[50] S. Vetrivel, J.S. Do, M.-Y. Cheng, B.J. Hwang, Simple catalyst for the effective growth of carbon nanotubes by CVD, J. Phys. Chem. C 111 (44) (2007) 16211-16218.

[51] M. Keidar, A. Shashurin, J. Li, O. Volotskova, M. Kundrapu, T.S. Zhuang, Arc plasma synthesis of carbon nanostructures: where is the frontier? J. Phys. D: Appl. Phys. 44 (17) (2011) 174006-174012.

[52] V.I. Perekrestov, A.S. Kornyushchenko, Yu.A. Kosminskaya, Formation of nanosystems under near-equilibrium copper condensation in an ultrapure inert medium, JETP Lett. 86 (12) (2008) 767-771.

[53] V.I. Perekrestov, Yu.O. Kosminska, A.S. Kornyushchenko, V.M. Latyshev, Selfassembly of porous Cu structures during steady-state condensation of weakly supersaturated vapors, J. Porous Mater. 21 (6) (2014) 1159-1167.

[54] H. Wang, Y. Huang, Z. Tan, X. Hu, Fabrication and characterization of copper nanoparticle thin-films and the electrocatalytic behavior, Anal. Chim. Acta 526 (1) (2004) 13-17.
[55] Z. Zhang, H. Che, Y. Wang, X. She, J. Sun, P. Gunawan, Z. Zhong, F. Su, Facile solvothermal synthesis of porous cubic Cu microparticles as copper catalysts for Rochow reaction, ACS Appl. Mater. Interfaces 4 (3) (2012) 1295-1302.

[56] Y. Isomura, T. Narushima, H. Kawasaki, T. Yonezawa, Y. Obora, Surfactant free single nano-sized colloidal $\mathrm{Cu}$ nanoparticles for use as an active catalyst in Ullmann-coupling reaction, Chem. Commun. 48 (2012) 3784-3786.

[57] Q. Jin, L. Zhang, H. Cao, T. Wang, X. Zhu, J. Jiang, M. Liu, Self-assembly of copper (II) ion-mediated nanotube and its supramolecular chiral catalytic behavior Langmuir 27 (22) (2011) 13847-13853.

[58] S. Siddhanta, Ch Narayana, Surface enhanced Raman spectroscopy of proteins: implications for drug designing, Nanomater. Nanotechnol. 2 (2012) 1-13.

[59] X. Zhang, Q. Zhou, Y. Huang, Z. Li, Z. Zhang, The nanofabrication and application of substrates for surface-enhanced Raman scattering, Int. J. Spectrosc. 2012 (2012) 1-8.

[60] G. Perny, B.L. Saint Martin, Influence des caractéristiques du plasma initiateur sur la formation des couches minces d'oxydes de cuivre dans la pulvérisation cathodique reactive, J. Phys. 25 (1964) 993-998.

[61] B.L. Saint Martin, G. Perny, M. Samirant, P. van den Berge, Aspects physicochimiques de la préparation de couches minces semiconductrices par condensation de plasmas froids, Rev. Phys. Appl. 1 (1966) 230-236.

[62] H.O. Pierson, Handbook of Chemical Vapor Deposition (CVD): Principles, Technology, and Applications, 2nd edition, Noyes Publications, New York (1999), p. 153-154.

[63] Y. Pauleau, A.Y. Fasasi, Kinetics of sublimation of copper(II) acetylacetonate complex used for chemical vapor deposition of copper films, Chem. Mater. 3 (1991) 45-50.

[64] A.A. Chernov, E.I. Givargizov, H.S. Bagdasarov, V.A. Kuznecov, L.N. Demyanec, A.N. Lobachev, in: B.K. Vainstein, A.A. Chernov, L.A. Shuvalov (Eds.), Modern Crystallography, vol. 3, Nauka, Moscow, 1980, pp. 7-232 (in Russian). 